\title{
DISCRIMINATIVE CHANNEL SELECTION METHOD FOR THE RECOGNITION OF ANTICIPATION RELATED POTENTIALS FROM CCD ESTIMATED CORTICAL ACTIVITY
}

\author{
G. Garipelli ${ }^{\dagger}$, R. Chavarriaga ${ }^{\dagger}$, F. Cincotti ${ }^{\ddagger}$, F. Babiloni ${ }^{\ddagger}$, J. del. R. Millán ${ }^{\dagger}$ * \\ ${ }^{\dagger}$ CNBI, School of Engineering \\ Ecole Polytechnique Fédérale de Lausanne \\ Station 11, CH-1015, Switzerland. \\ ${ }^{\ddagger}$ Laboratory of Neuroelectrical Imaging and BCI \\ Fondazione Santa Lucia IRCCS \\ Via Ardeatina 306, Roma 00179, Italy.
}

\begin{abstract}
Recognition of brain states and subject's intention from electroencephalogram (EEG) is a challenging problem for braincomputer interaction. Signals recorded from each of EEG electrodes represent noisy spatio-temporal overlapping of activity arising from very diverse brain regions. However, un-mixing methods such as Cortical Current Density (CCD) can be used for estimating activity of different brain regions. These methods not only improve spatial resolution but also signal to noise ratio, hence the classifiers computed using this activity may ameliorate recognition performances. However, these methods lead to a multiplied number of channels, leading to the question - "How to choose relevant and discriminant channels from a large number of channels?". In the current paper we present a channel selection method and discuss its application to the recognition of anticipation related potentials from surface EEG channels and CCD estimated cortical potentials. We compare the classification accuracies with previously reported performances obtained using $\mathrm{Cz}$ electrode potentials of 9 subjects ( 3 experienced +6 naïve). As hypothesised, we observed improvements for most subjects with channel selection method applied to CCD activity as compared to surfaceEEG channels and baseline performances. This improvement is particularly significant for subjects who are naïve and did not show a clear pattern on ERP grand averages.
\end{abstract}

\section{INTRODUCTION}

Recognition of subject's intention on single trials from electroencephalogram (EEG) for brain-computer interaction is a challenging problem. EEG represents noisy spatio-temporal overlapping of activity arising from very diverse brain regions, i.e., a single scalp electrode picks up the mixed temporal activity of myriads of neurons at very different brain areas. Previous studies show that un-mixing methods are

\footnotetext{
*Authors thanks the European IST program project BACS (FP6-IST027140) and Neuromath for supporting this work. Thanks also Dr. P. Ferrez, L. Uldry and N. Bourdaud for their valuable suggestions.
}

likely to improve the signal to noise ratio (SNR) which helps in computing reliable classifiers $[1,2,3]$. Cortical Current Density (CCD) inverse method is one such a method based on distributed linear inverse estimation of a dipole activity whose orientation is assumed to be perpendicular to a small triangular patch of cortical mantle $[2,4,5]$. However, un-mixing leads to a multiplied number of channels (dipole activities) leading to the question -"How to chose relevant and discriminant channels from a large number of available channels?". In the current paper we present our channel selection method based on discriminability of features and discuss its application to the recognition of anticipation related potentials from surface EEG and CCD estimated cortical activity.

Anticipation is a cognitive state of a subject, reflecting expectation of future events based on past events. In EEG this state appears mainly as an increasing negativity under the site of vertex electrode $(\mathrm{Cz})$ which can be interpreted as pre-activation of neural substrates involved in executing actions on the occurrence of events in future $[6,7,8]$. In our previous studies, we argued that single trial recognition of anticipation related potentials can be exploited for BrainComputer Interaction (BCI) [9, 10], and in a recent pilot experiment we demonstated a prototype of anticipation based BCI (aBCI) [11]. These studies were based on features computed using activity of the $\mathrm{Cz}$ electrode alone. However, as the negativity spreads around central electrodes, features of these potentials can be observed from the signals of surrounding electrodes which may carry discriminable features that could be useful for improving classification performance. In addition, the potentials of all the electrodes can be used to un-mix the cortical dipole activity and a specific subset of the dipole activity can be used for calculating more reliable classifiers. However, for calculating classifiers using surface EEG or cortical activity, we need a systematic procedure for choosing channels which are the most discriminant.

In the current paper we explore features selected from surface EEG channels as well as from CCD estimated cortical activity, and compare classification accuracies to base- 
line accuracies achieved with $\mathrm{Cz}$ electrode features. The current paper is organized as follows-In section 2 we introduce the experimental paradigm to record anticipation related potentials as well as the pre-processing applied to EEG signals. In the same section we describe EEG grand averages showing the presence of anticipation related features in electrodes apart from $\mathrm{Cz}$. In section 3 we briefly describe CCD inverse method and present cortical localization of anticipation related potentials. In the same section, we present feature selection and a channel selection method and classification technique used in the current study. In section 4 we compare the accuracies achieved with the application of channel selection to surface EEG channels and CCD estimated cortical activity to the baseline accuracy obtained with single electrode features. Finally, in section 5 we discuss the results.

\section{EXPERIMENT}

To record anticipation related potentials we have used a classical Contingent Negative Variation (CNV) paradigm [8, 7]. In this paradigm a warning stimulus (S1) predicts the appearance of an imperative stimulus (S2) in a fixed interstimulus-interval (ISI). For simulating anticipatory and nonanticipatory behaviors we used a CNV paradigm with relevant (GO) and irrelevant (NOGO) conditions ( See Fig. 1 for the $\mathrm{CNV}$ paradigm used in the current study and refer [10] for more details).

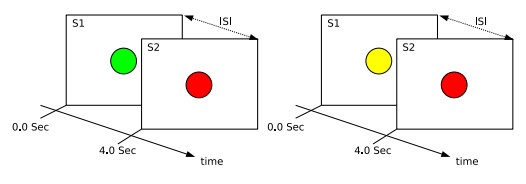

(a)

(b)

Fig. 1. The CNV experimental setup. An warning stimulus (S1) at time $t=0 s$ is displayed and then an imperative stimulus (S2) with a red dot on the screen is presented with ISI of 4s. (a) the GO condition is represented by a green flash for $\mathrm{S} 1$ and subjects are instructed to anticipate and press a button as soon as $\mathrm{S} 2$ is presented. (b) the NOGO condition is represented $\mathrm{S} 1$ by a yellow dot. The subjects are instructed to do nothing for this condition.

\subsection{Data acquisition and prepossessing}

The EEG signals were acquired for 9 subjects using 32 (subjects 4, 5 and 9) or 64 (remaining 6 subjects) electrodes according to the $10 / 20$ international system with a sampling rate of $512 \mathrm{~Hz}$ in 4 sessions with 50 trials in each session with equi-probable GO and NOGO conditions. Raw EEG signals were first spatially filtered by using a common average reference (CAR), then filtered using a low-pass filter with cut off frequency of $15 \mathrm{~Hz}$. The trials were extracted and separated into GO and NOGO trials using $\mathrm{S} 1$ as the reference with [-1.0 5.0]s as total trial interval. Baseline is computed using window of $\left[\begin{array}{ll}-1 & 0\end{array}\right] \mathrm{s}$.

\subsection{ERP grand averages}

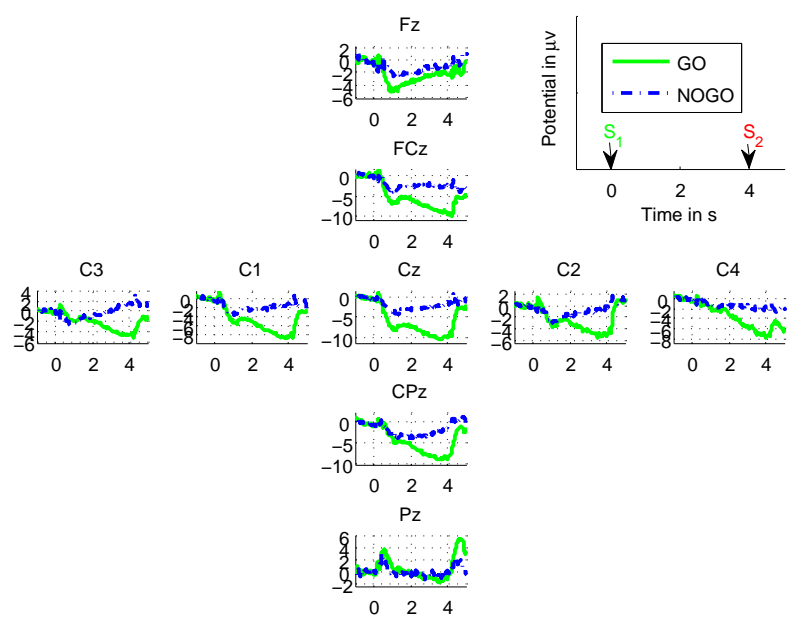

Fig. 2. ERP grand average of 6 subjects for GO and NOGO conditions at different electrode sites (reported for 64 electrode EEG setup; Similar observations are made for 32 electrode setup)

The EEG grand averages as computed over subjects for GO and NOGO conditions show clear differences (see Fig. 2). An evoked response due to $\mathrm{S} 1$ is observed in around $0.3 \mathrm{~s}$ to $0.4 \mathrm{~s}$, in both conditions. The potential at $\mathrm{Cz}$ during GO condition is composed of an early peak around 1s and a late peak between $3.5 \mathrm{~s}$ and $4.0 \mathrm{~s}$ which is consistent with previous studies [7]. Both components are atributed to alertness and expectancy with motor preparation as an extra component in the late peak. Although both components are discriminable at the $\mathrm{Cz}$ electrode activity, the early and late components are also visible in other electrodes (see Fig. 2). The early component is prominently discriminable in the fronto-central electrodes $(\mathrm{Fz}, \mathrm{FCz}$, and $\mathrm{Cz}$ ), which is likely due to subject's attentiveness to the warning stimuli [7]; while the late component is prominent in the central (C1, C2, C3, C4, FCz, Cz and CPz) which is likely due to subjects' motor preparation [7]. It is worth noting that the subjects considered in the current study were naïve except for subjects 1, 2 and 5 who had a little experience with the protocol. From the grand averages of each subject's potentials at $\mathrm{Cz}$ electrode as shown in the Fig. 3, it is also clear that each individual has a different evolution of CNV potential and is more discriminable from the NOGO condition for the experienced subjects than for the naïve subjects. 

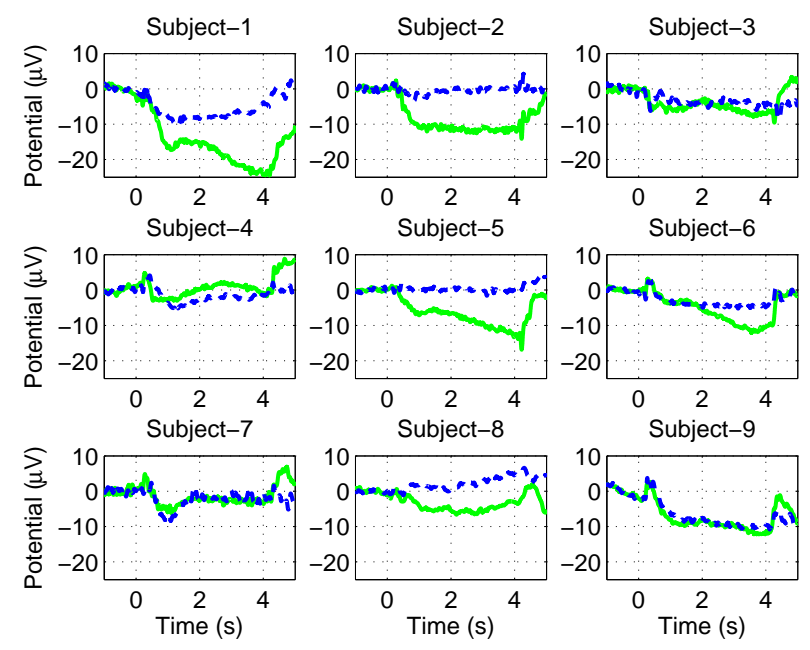

Fig. 3. The ERP grand averages computed for potentials at $\mathrm{Cz}$ electrode separately for 9 subjects (solid green line for the GO condition and broken line for the NOGO condition).

\section{METHODS}

In this section we introduce briefly the CCD inverse method followed by channel selection method to obtain the most discriminable channels and the classification method used in the current study.

\subsection{Estimated cortical activity}

EEG signals represent the noisy spatio-temporal overlapping of activity arising from very diverse brain regions, i.e., a single scalp electrode picks up the mixed temporal activity of myriads of neurons at very different brain areas. Unmixing methods are likely to improve the signal to noise ratio (SNR) which could help in computing reliable classifiers. These methods are under determined and lead to non-unique solutions. However, using constraints based on biophysical properties of the neural tissue and a head model lead to a unique and realistic solution.

The CCD inverse method is based on distributed linear inverse estimation of a dipole activity whose orientation is assumed to be perpendicular to a small triangular patch of cortical mantle. The shape of the cortical mantle is divided into 3013 triangular patches (vortices) and is obtained using an average head model. Since this estimation is based on weighted minimum-norm, it forces the dipoles to comprehend the recorded EEG signals with minimum energy without penalizing the deeper sources (see $[2,4,5]$ for more details).

Fig. 4 shows topographic representations of the ERP grand averages of the estimated cortical activity using CCD inverse method for GO and NOGO conditions at the early

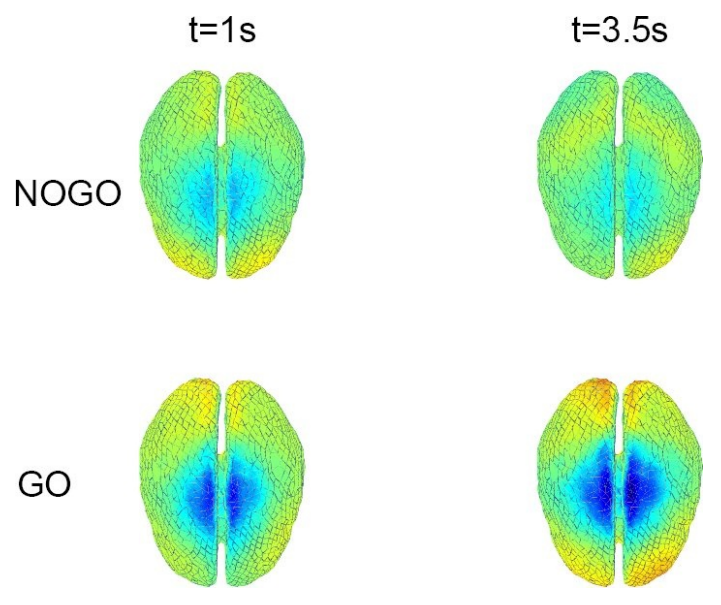

(a)

Fig. 4. Topographic maps of the estimated cortical activity for GO and NOGO trials obtained using the CCD inverse of EEG grand averges (computed using EEG of six subjects recorded with 64 electrode configuration; we removed outer electrodes, that are prone to artifacts, in computing CCD inverse matrix) shown at the time of early-peak (time $1.0 \mathrm{~s}$ ) and of late-peak (at time $3.5 \mathrm{~s}$ ) of the CNV potential (the orientation of the nose is to the bottom)

peak and late peak timings. It can be observed that the negativity of the dipoles increases in the fronto-central and centro-parietal areas in both hemispheres. The early component appears in the fronto-central areas and, as time progresses, the negativity spreads over central and centro-parietal areas. This observation reinforces the idea that the activity due to anticipatory behavior is not focused in one area and the use of features computed from the activity from multiple sources is likely to improve the classification accuracies. However, the projection of surface EEG channel data to CCD estimated dipole activity results in a dramatic increase in the number of available channels. This leads to the problem of choosing the most discriminable channels among all the available channels.

In this following subsections we address the question"Given the signals from a large number of channels, how to choose the best $K$ channels for calculating classifiers?". To address it we first perform feature selection as explained in the following paragraphs.

\subsection{Feature selection}

Since the CNV potential exhibits a slowly increasing negativity, it is usually characterized by its slope $[12,7]$. In the current paper instead of the slope alone, we propose to use higher order features such as coefficients of least square estimated polynomial $\left(\alpha_{i} ; \tilde{v}_{C z}=\alpha_{0}+\alpha_{1} t^{1}+\ldots+\right.$ 
$\left.\alpha_{n} t^{n}\right)$. Each trial is then described by the feature vector $s=\left[\begin{array}{lllll}\alpha_{0} & \alpha_{1} & \alpha_{2} & \ldots & \alpha_{n}\end{array}\right]^{T}$. To find the best polynomial order $(n)$ for a given subject we first explore orders in the range $1,2 \ldots 6$ and compare the channel discriminant power $\left(\rho^{c}\right)$ using Cz electrode activity as follows (note thats, $n=1$ means that the signal is approximated with a line and features are the slope and the offset of the line).

For a given feature, $f$ (polynomial coefficient in our case), in a two-class scenario the $\rho$ is calculated as the portion of samples lying in the non overlapping zones between boundaries of each class. Being $f_{1}$ and $f_{2}$ are features for each class, $\rho$ is given by,

$$
\rho(f)=\frac{\eta_{f 1}+\eta_{f 2}}{N_{t 1}+N_{t 2}}
$$

where, $\eta_{f 1}$ and $\eta_{f 2}$ are the number of discriminant samples of each class located in non-overlapping zones, given by,

$\eta_{f 1}=\sum_{i=1}^{N t_{1}}\left(1\left(s_{f 1}(i)>\max \left(s_{f 2}\right)\right)+1\left(s_{f 1}(i)<\min \left(s_{f 2}\right)\right)\right)$

$\eta_{f 2}=\sum_{i=1}^{N t_{2}}\left(1\left(s_{f 2}(i)>\max \left(s_{f 1}\right)\right)+1\left(s_{f 2}(i)<\min \left(s_{f 1}\right)\right)\right)$

where, $N t_{1}$ and $N t_{2}$ are number of trials of each class, $s_{f 1}$ and $s_{f 2}$ are vectors of feature $f$ of class 1 and 2 . And $\min \left(s_{f k}\right)$ and $\max \left(s_{f k}\right)$ defines the boundaries of feature distribution of $k^{t h}$ class, and $1(x)$ is function defined by

$$
1(x)=\left\{\begin{array}{l}
1 \text { if } x \text { is true } \\
0 \text { otherwise }
\end{array}\right.
$$

The $\rho$ is a value in $[01]$, represents non-overlapping region in the sample distribution of that feature for the two classes. If $\rho=0$, it means the distributions are fully overlapped and $\rho=1$ means no overlap (see [3] for more details). The above formula is devised with the assumption that the features follow unimodal distribution. However, we pruned the feature set to remove the outliers from the distribution with a confidence interval of $[\mu-1.77 . \sigma ; \mu+1.77 . \sigma]$ (this range is chosen arbitrarily). The discriminant power of the channel, $\rho^{c}$, is computed as the mean $\rho$ of all the features (coefficients). The best polynomial order for a given subject is chosen based on the highest $\rho^{c}$ computed using the $\mathrm{Cz}$ electrode alone. Once the best order is selected for a subject it is kept constant for the next step where we chose the best channels.

\subsection{Channel selection}

The channel selection in the case of EEG refers to choosing best $K$ channels from 21 or 41 electrodes for the 32 and 64 electrode setup respectively (we removed the peripheral electrodes to avoid artifacts). The channel selection in the case of CCD vortices is choosing the best $K$ channels from 3013 vortices. For both cases we compute $\rho^{c}$ for each channel using the best polynomial order obtained in the previous step. We sort the channels based on the $\rho^{c}$ value and then chose channels that have $\rho^{c}$ in the range of $\left[\frac{(100-P) \cdot \rho^{c}}{100} \rho^{c}\right]$. For example if the highest $\rho^{c}$ is 0.8 and if $P=10 \%$ then we choose the channels whose $\rho^{c}$ are in the range of [0.72 0.8$]$ for building the classifier.

\subsection{Classification}

Once the best $K$ channels are chosen based on the channel selection procedure as explained above, we train a linear discriminant analysis (LDA) classifier [13]. The feature vector is the concatenation of the coefficients of the best polynomial approximation for the selected channels. We treat the features as independent (i.e., we constrained to diagonal covariance matrices in finding projection matrix for LDA) to avoid the problem of over-fitting which arises due to large number of features compared to the number of trials. We then compare classification accuracies obtained with different values of $P$. The results of this comparison are explained in detail in the next section.

\section{RESULTS}

Since the subjects were informed to press a button on the arrival of S2 (i.e., after 4 s) as explained in the section 2, we computed features using potentials recorded up to $3.5 \mathrm{~s}$ after the onset of S1 so as to avoid any movement related potential that could contaminate the anticipation process [7]. To asses the generalization capabilities over time, we used the first three sessions as training data and the last session as test data. This way of splitting EEG data yields a better unbiased estimate of the generalization capabilities of a classifier than, e.g., one-leave-out due to non-stationarity of brain signals. In following paragraphs we compare the classification accuracies of channel selection method applied to EEG electrodes and CCD estimated cortical activity to baseline accuracies.

Firstly, we computed the baseline accuracies using the best order given by the feature selection method explained in the section 3.2 using the potentials recorded using $\mathrm{Cz}$ electrode. Table. 1 shows the best orders and corresponding baseline accuracies. For most of the subjects the best order is 1 (i.e. using slope and offset as features). Although the baseline accuracies for most of the subjects are close to chance level, for subjects 1,2 and 5 (with some experience with protocol) the classification accuracies are $69.4 \%$, $81.6 \%$ and $65.3 \%$ respectively, are far from chance level.

Secondly, we applied channel selection method as explained in section 3.3 to EEG electrodes and computed clas- 
Table 1. Baseline performances: The best order and test accuracies using the features of $\mathrm{Cz}$ electrode features.

\begin{tabular}{|c|c|c|}
\hline Subject & Best order & Accuracy (\%) \\
\hline $\mathbf{1}$ & 1 & 69.39 \\
$\mathbf{2}$ & 1 & 81.63 \\
$\mathbf{3}$ & 1 & 41.67 \\
$\mathbf{4}$ & 1 & 42.00 \\
$\mathbf{5}$ & 1 & 65.31 \\
$\mathbf{6}$ & 1 & 50.00 \\
$\mathbf{7}$ & 1 & 48.00 \\
$\mathbf{8}$ & 1 & 45.83 \\
$\mathbf{9}$ & 3 & 44.00 \\
\hline
\end{tabular}

sifiers for different values of $P$. We compare the classification accuracies with baseline accuracies separately for each subject in Fig. 5. As it can be seen from the figure, for six subjects $(3,4,6,7,8$ and 9) this method significantly improved the accuracies compared to the baseline. However, for the remaining subjects the accuracies are close to the baseline. The number of selected channels, $K$, for each subject for different values of $P$ are shown in Fig. 6. The number of channels increase exponentially after a certain value of $P$ for most of the subjects. However, for subject 5 the selected channels does not vary and stays at one and is $\mathrm{Cz}$ electrode. This is due to the reason that $\mathrm{Cz}$ electrode for this particular subject is far better discriminable than any other electrode.

Thirdly, we applied channel selection method to CCD estimated cortical activity and obtained classification accuracies for different values of $\mathrm{P}$ as shown in the Fig. 5. From the figure it is evident that for all the subjects except subject 2 and 8 there is a significant improvement with CCD method compared to the baseline accuracies. This improvement is particularly significant for the subjects $3,4,6,7$ and 9 who have not produced a clear CNV potential on the grand averages (see Fig. 3) and the baseline accuracies are close to chance level. The channel selection method applied to CCD vortices lead to better accuracies for most of the subjects (except subject 2 and 8 ) in comparison to EEG electrodes. The number of selected vortices as function of $\mathrm{P}$ is exponential for all the subjects (see Fig. 6).

\section{DISCUSSION}

Signals recorded using surface EEG is a noisy spatio-temporal mixture of cortical sources. Un-mixing methods based on biophysical properties can be used for improving not only spatial resolution but also SNR which can be helpful for computing classifiers for BCI. Previous studies reported improvement in classification accuracies with the use of linear
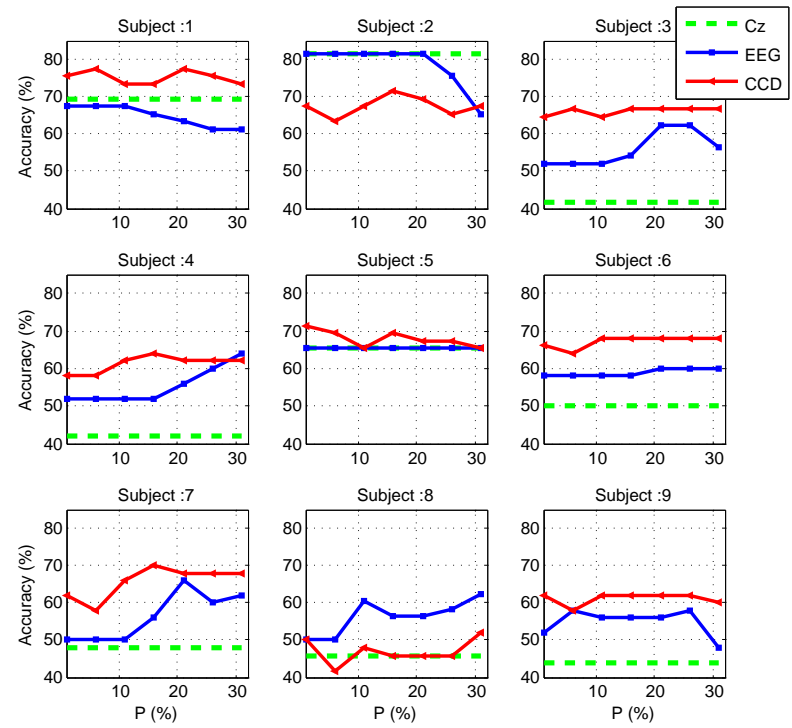

(a)

Fig. 5. Comparison of classification accuracies obtained for $\mathrm{Cz}$ electrode features with that of channel selection for EEG electrodes and CCD vertexes for different thresholds of $P$
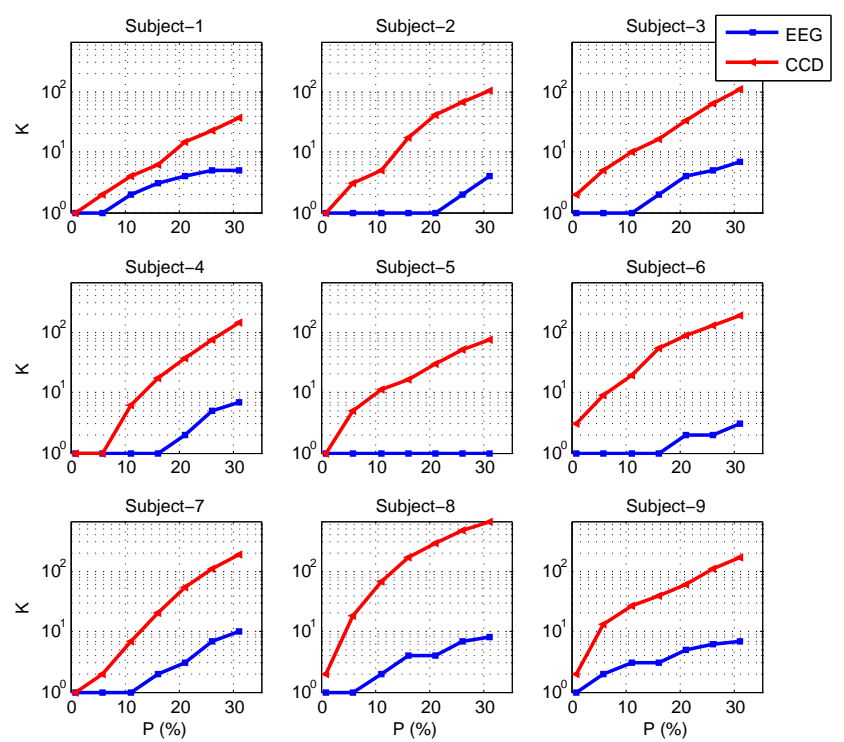

Fig. 6. Number of channels selected $(K)$ for EEG (blue line with squares) and CCD (red line with triangles) for different thresholds of $P$

inverse methods for the classification mental imagery $[1,2]$ and cognitive states [3]. In the current paper we studied the use of CCD inverse method for the recognition of anticipation related potentials which are shown to be exploited for BCI $[9,10,11]$. One of the challenges in working with es- 
timated cortical potentials is that the number of available channels get multiplied drastically and a method for choosing a subset of them for calculating classifiers is essential. In the current paper we address this issue by using a channel selection scheme that selects channels with most discriminant power. We tested this scheme with surface EEG channels (21 or 41 channels) as well as CCD estimated cortical activity (3013 channels).

Comparing the accuracies obtained with the EEG channel selection to baseline accuracies obtained with $\mathrm{Cz}$ electrode potentials we observe that for most subjects there is an improvement (except for three subjects), suggesting that using multi-electrode features may lead to more robust classifiers. Furthermore, the use of the CCD inverse method results in a significant improvement over baseline for most subjects. This improvement is particularly significant for the subjects whose baseline accuracy is close to chance level (see Fig. 5) and the CNV potential on ERP grand averages is not so clear (see Fig. 3). The variation in accuracy across the subjects is likely due to the fact that subjects 1,2 and 5 are slightly experienced with the protocol beforehand whereas the other subjects are completely naïve. The CCD vortex selection based classification outperforms the EEG electrode selection for most of the subjects suggesting that the CCD inverse method improves SNR leading to better discriminable features than EEG.

Recently it has been demonstrated on-line operation of a BCI system using CCD inverse technique [2]. It was feasible due to the fact that most of the computation is performed during offline stage and on-line processing requires just one matrix multiplication. Presented results show that for some subjects, CCD-based classification performance is at least as good as classifiers based on a single electrode $(\mathrm{Cz})$. Moreover, it has been hypothesized that experience allows subjects to better modulate these signals $[9,11,12]$. Taking this into account, we can devise a scheme were classification is initially based on inverse solutions, and if signals are observed to have a better separability (e.g. through subject training), the $\mathrm{BCI}$ can switch to a lighter $\mathrm{Cz}$ featurebased classifier.

\section{REFERENCES}

[1] M. Congedo, F. Lotte, and A. Lécuyer, "Classification of movement intention by spatially filtered electromagnetic inverse solutions.," Phys Med Biol, vol. 51, no. 8, pp. 1971-1989, Apr 2006.

[2] F. Cincotti, D. Mattia, F. Aloise, S. Bufalari, L. Astolfi, F. De V. Fallani, A. Tocci, L. Bianchi, M. G. Marciani, S. Gao, J. del. R. Millán, and F. Babiloni, "High-resolution EEG techniques for brain-computer interface applications.," J Neurosci Methods, vol. 167, no. 1, pp. 31-42, Jan 2008.
[3] P. W. Ferrez, Error-related EEG potentials in braincomputer interfaces., Ph.D. thesis, EPFL, 2007.

[4] F. Babiloni, C. Babiloni, L. Locche, F. Cincotti, P. M. Rossini, and F. Carducci, "High-resolution electroencephalogram: Source estimates of Laplaciantransformed somatosensory-evoked potentials using a realistic subject head model constructed from magnetic resonance images.," Med. Biol. Eng. Comput., vol. 38, no. 5, pp. 512-519, Sep 2000.

[5] F. Babiloni, F. Cincotti, C. Babiloni, F. Carducci, D. Mattia, L. Astolfi, A. Basilisco, P. M. Rossini, L. Ding, Y. Ni, J. Cheng, K. Christine, J. Sweeney, and B. He, "Estimation of the cortical functional connectivity with the multimodal integration of highresolution EEG and fMRI data by directed transfer function.," Neuroimage, vol. 24, no. 1, pp. 118-131, Jan 2005.

[6] G. J. M. Van Boxtel and K. B. E. Böcker, "Cortical measures of anticipation," Journal of Psychophysiology, vol. 18, pp. 61-76, 2004.

[7] B. Rockstroh, T. Elbert, A. Canavan, W. Lutzenberger, and N. Birbaumer, Slow Cortical Potentials and Behaviour, Urban \& Schwarzenberg, 2nd edition, 1989.

[8] W. G. Walter, R. Cooper, V. J. Aldridge, W. C. McCallum, and A. L. Winter, "Contingent negative variation: An electric sign of sensorimotor association and expectancy in the human brain," Nature, vol. 203, pp. 380-4, 1964.

[9] G. Garipelli, R. Chavarriaga, and J. del. R. Millán, "Recognition of anticipatory behavior from human EEG.," in Proc 4th Intl. Brain-Computer Interface Workshop and Training Course, Graz, 2008.

[10] G. Gangadhar, R. Chavarriaga, and J. del. R. Millán, "Fast recognition of anticipation related potentials," IEEE Tran Biomed Eng, vol. 56, no. 4, pp. 1257-60, 2009.

[11] G. Garipelli, R. Chavarriaga, and J. del. R. Millán, "Anticipation based brain-computer interfacing (aBCI)," in Proc 4th Intl. IEEE EMBS Conference on Neural Engineering, Antalya, 2009.

[12] L. Bozinovska, S. Bozinovski, and G. Stojanov, "Electroexpectogram: Experimental design and algorithms," in Proceedings IEEE Biomedical Engineering Days, 1992, 1992, pp. 58-60.

[13] R. O. Duda, P. E. Hart, and D.G. Stork, Pattern Classification, Wiley \& Sons, 2nd edition, 2001. 